Revista Brasileira de Meteorologia, v.27, n.2, 219 - 228, 2012

\title{
SOBRE O SINAL DE UM DOWNSCALING DINÂMICO ÀS OSCILAÇÕES INTRASSAZONAIS DE PRECIPITAÇÃO NO SETOR NORTE DO NORDESTE DO BRASIL
}

\author{
JOSÉ MARIA BRABO ALVES ${ }^{1}$, EVERALDO BARREIROS DE SOUZA ${ }^{2}$, ALEXANDRE ARAÚJO \\ COSTA $^{3}$, EDUARDO SÁVIO PASSOS RODRIGUES MARTINS ${ }^{1}$ E EMERSON MARIANO DA SILVA ${ }^{3}$ \\ ${ }^{1}$ Fundação Cearense de Meteorologia e Recursos Hídricos (FUNCEME), Fortaleza, CE, Brasil \\ ${ }^{2}$ Universidade Federal do Pará (UFPa), Faculdade de Meteorologia, Belém, PA, Brasil \\ ${ }^{3}$ Universidade Estadual do Ceará (UECE), Departamento de Física, Fortaleza, CE, Brasil
}

brabo@funceme.br, everaldo@ufpa.br, alexandrearaujoc@gmail.com,esm9@funceme.br, emerson@uece.br

Recebido Fevereiro de 2010 - Aceito Dezembro de 2011

\begin{abstract}
RESUMO
Oscilações intrassazonais são fatores controladores da variabilidade pluviométrica interanual de áreas tropicais. O conhecimento de como os modelos numéricos reproduzem suas variabilidades é importante para entender melhor suas atuações e subsidiar operações de previsão de tempo e clima. Neste artigo investiga-se a sensibilidade de um modelo de downscaling dinâmico de precipitação na reprodução das oscilações intrassazonais observadas no setor norte do Nordeste do Brasil (SNNEB - $45^{\circ} \mathrm{W}-37^{\circ} \mathrm{W}$ e $2^{\circ} \mathrm{S}-12^{\circ} \mathrm{S}$ ) no período de 1974 a 2000 . Os resultados mostraram que a precipitação simulada sobre o SNNEB explicou aproximadamente $70 \%$ da variabilidade da precipitação observada no trimestre fevereiro a abril nesta região. Estatísticas de médias, desvios normalizados e percentuais neste trimestre, nessa região, em anos de contrastes climáticos nos Oceanos Pacífico e Atlântico Tropicais, também foram bem capturadas pela precipitação simulada através do downscaling. Análises espectrais com uso de ondeletas mostraram que o downscaling dinâmico tem potencial para reproduzir picos espectrais de precipitação observada no SNNEB nas escalas acima de 8 dias, e nas escalas entre 64 e 128 dias para o período de 01 de janeiro a 30 de junho.
\end{abstract}

Palavras-chave: intrassazonal, precipitação, ondeletas, análise espectral.

\begin{abstract}
ON THE INTRASEASONAL SIGN OF THE RAINFALL OVER NORTHERN NORTHEAST BRAZIL SIMULATED BY A DYNAMIC DOWNSCALING. Intraseasonal oscillations are factors controlling the interannual variability of tropical rainfall. The knowledge of how numerical models reproduce its variability is important to better understand their roles and support operations of weather and climate. This paper investigates the sensitivity of a downscaling dynamic precipitation model in predicting the intraseasonal oscillations observed in the northern sector of Northeast Brazil (SNNEB $-45^{\circ} \mathrm{W}-37^{\circ} \mathrm{W}$ and $2^{\circ} \mathrm{S}-12^{\circ} \mathrm{S}$ ) during the period from 1974 to 2000 . The results showed that rainfall was simulated on SNNEB explained more than $70 \%$ of the variability of rainfall observed in the quarter February to April in this region. Statistical of averages, standard deviations and percent this quarter, in this region in years of climatic contrasts in the Oceans Pacific and Atlantic Tropical were also well captured by the precipitation simulated by downscaling. Spectral analysis with the use of wavelets showed that dynamic downscaling has the potential to reproduce observed spectral peaks of precipitation in SNNEB scales up to 8 days and the scales between 64 and 128 days for the period January 1 to June 30 .
\end{abstract}

Keywords: Intraseasonal rainfall, wavelets, spectral analysis.

\section{INTRODUÇÃO}

Oscilações Intrasasonais Tropicais (OIT), definidas por alguns pesquisadores entre 10-90 dias, têm significantes papéis na variabilidade interanual da precipitação em muitas regiões do globo, e envolvem importantes mecanismos de variabilidades de baixas frequências no sistema climático, principalmente nos trópicos (Rasmusson e Arkin, 1993; Jones et al., 2004). Nesta escala, a Oscilação de Madden Julian (OMJ) é um modo dominante, sendo mais ativa no inverno austral (Madden e 
Julian, 1994), cujo ciclo de vida ao redor do globo dura em torno de 30 a 60 dias (Weickmann, 1991; Madden e Julian, 1994). Segundo Jones et al. (2004), quando se refere às ocorrências no inverno austral, defini-se como sendo a OMJ, e quando estas ocorrências se dão no verão austral são denominadas de OIT.

As influências da OMJ e da OIT nos padrões de precipitação nos trópicos e extratrópicos do globo foram amplamente documentadas. Por exemplo, OMJ e OIT influenciam eventos de precipitação em regiões de Monções na Ásia-Austrália e moderadamente nas Américas do Norte e Sul (Jones, 2000; Mo, 2000; Paegle, et al., 2000; Higgins e Shi, 2001; Jones e Carvalho, 2002; Carvalho et al., 2004). Para precipitação diária na Califórnia, Jones (2000) mostrou em seus resultados que OMJ está associada com eventos extremos de precipitação. Os resultados de Mo (2000) evidenciaram que a precipitação de Monção, sobre o Arizona e Novo México, associa-se a eventos de precipitação que se propagam para leste desde o Pacífico Norte. Os períodos mais úmidos e secos nesta região são modulados por um modo que compreende de 22-25 dias. Paegle et al. (2000), usando análise espectral, mostraram que a convecção sobre a América do Sul tem um modo dominante relatado a OMJ, principalmente o padrão de dipolo dos centros de convecção associados a da Zona de Convergência do Atlântico Sul (ZCAS) e a região do alti-plano subtropical. Higgins e Shi (2001) mostraram que a variabilidade da precipitação pentadal de verão na região de Monção da América do Norte está associada à influência da OMJ.

Jones e Carvalho (2002) mostraram que há dois regimes distintos de precipitação na região de Monção da América do Sul, associados à OMJ, com escala temporal de 10 a 70 dias. $\mathrm{O}$ regime de ventos de oeste, com ventos cruzando o equador na direção sudoeste, transportando umidade da Amazônia, em baixos níveis fechando com uma circulação ciclônica anômala na costa da Argentina e Uruguai. Incremento de convecção e precipitação é observado no centro oeste e sudeste do Brasil. Supressão de convecção e precipitação ocorre no Alti-Plano Boliviano e setor norte da América do Sul. Para o regime de ventos de leste, as características são contrárias às observadas no regime de ventos de oeste. Carvalho et al. (2004) mostraram evidências de que a OMJ modula a intensidade da ZCAS. A OMJ associa-se a intensa ZCAS com períodos de duração maior do que três dias de atuação. Na fase positiva da OMJ, há uma supressão de convecção na região da Indonésia e aumento no Pacífico Central e no Nordeste da América do Sul.

Especificamente para o Nordeste do Brasil (NEB), destacam-se os estudos de Repelli et al. (1998), que mostraram a atuação da $\mathrm{OMJ}$ na distribuição de precipitação na segunda quinzena de março de 1997, causando chuvas intensas em todo a região. Nobre e Melo (2001) analisaram as variações da precipitação no período de 1998-2000 no setor norte do NEB, as quais foram fortemente influenciadas pela OMJ. Neste período foram marcantes as oscilações de precipitação mais intensa e escassa no Nordeste do Brasil, com períodos de tempo típicos da OMJ (30 a 60 dias).

Downscaling climático tem sido executado desde o início da década de 80 , com maior ênfase maior durante os anos 90 e 2000. Para o Nordeste do Brasil destaca-se o estudo de Nobre et al. (1998) que mostrou que um aninhamento entre o ECHAM4.5 e o modelo regional espectral do NCEP/ NCAR (National Center for Environment Prediction-National Center for Atmospheric Research) que reproduziu algumas características associadas a Zona de Convergência Intetropical (ZCIT) junto a costa norte do NEB, que não foram detectadas pelo global ECHAM4.5. Mais recentemente, Sun et al. 2005 e 2006 avaliaram mais, detalhadamente, os resultados de uma simulação 1971-2000 e previsão 2001-2004 de um downscaling dinâmico com ênfase a precipitação sazonal para o NEB. Seus resultados mostraram que o downscaling climático tem potencial para ser usado como ferramenta de previsão sazonal de precipitação no NEB, principalmente seu setor norte.

Relacionado, a avaliação do sinal intrassazonal associado à OMJ nos dados de precipitação do NEB e sua comparação com modelagem dinâmica obtida via downscaling, praticamente nenhum estudo foi realizado. Assim sendo, o presente trabalho tem como objetivo identificar e comparar as características de oscilações intra-sazonais de precipitação observadas e simuladas por um dowscaling dinâmico (1974-2000) no NEB.

\section{MATERIAIS E MÉTODOS}

\subsection{Dados}

Foram utilizados dados diários de precipitação, derivados de um conjunto de estações pluviométricas distribuídas sobre o NEB (Figura 1), provenientes das redes observacionais pertencentes ao Instituto Nacional de Meteorologia, Agência Nacional de Águas e Núcleos Estaduais de Meteorologia e Recursos Hídricos, cobrindo o período de 1974-2000. Estes dados foram interpolados espacialmente, usando a técnica do inverso do quadrado da distância, para uma grade regular com resolução de $1^{\circ}$ em latitude e longitude (aproximadamente 111 $\mathrm{km}$ ). Detalhes sobre os procedimentos de controle de qualidade destes dados, bem como comparações com outras bases de dados encontram-se descritos em Souza et al. (2004).

Para este mesmo período, 1974-2000, utilizaram-se também as precipitações diárias simuladas pelo downscaling dinâmico (modelo regional espectral - RSM aninhado ao modelo global ECHAM4.5) numa grade regular de $60 \mathrm{~km}$ de resolução horizontal. 
Com a finalidade de se realizar comparações objetivas, foram geradas as séries temporais diárias da precipitação observada e simulada numa região representativa do setor norte do NEB (SNNEB), no domínio entre $45^{\circ} \mathrm{W}-37^{\circ} \mathrm{W}$ e $12^{\circ} \mathrm{S}-2^{\circ} \mathrm{S}$ em destaque na Figura 1. Estas séries foram construídas para os meses de janeiro a junho, período que compreende o semestre mais chuvoso do SNNEB (fevereiro a abril).

\subsection{Downscaling dinâmico}

O sistema de downscaling dinâmico de previsão climática sazonal para o NEB encontra-se descrito em Sun et al. (2005) e consiste do modelo regional espectral (RSM), em operação no NECP/NCAR (Juang e Kanamitsu, 1994), o qual foi aninhado ao modelo global de circulação geral da atmosfera (MCGA) ECHAM4.5 do Max-Planck Institute. O modelo global ECHAM4.5 possui resolução T42L19, truncamento triangular que corresponde a uma resolução horizontal de aproximadamente $2,8^{\circ}$ de latitude e longitude com dezenove níveis na vertical (Rockner et al., 1996). O sistema de downscaling RSM e ECHAM4.5 encontra-se em operação executando previsão sazonal de precipitação na FUNCEME desde 2001, com uma grade horizontal de $60 \mathrm{~km}$ e dezoito níveis na vertical, cobrindo o domínio tropical entre $23^{\circ} \mathrm{S}-16^{\circ} \mathrm{N}$ e $53^{\circ} \mathrm{W}-3^{\circ} \mathrm{E}$. As simulações sazonais neste downscaling consistiram de integrações de seis meses, entre 01 de Janeiro a 30 de Junho, de cada ano durante o período de 1971 a 2000. Estas simulações foram realizadas usando dez condições iniciais distintas fornecidas pelo ECHAM4.5, sendo que as variáveis componentes zonal e meridional do vento, umidade específica,

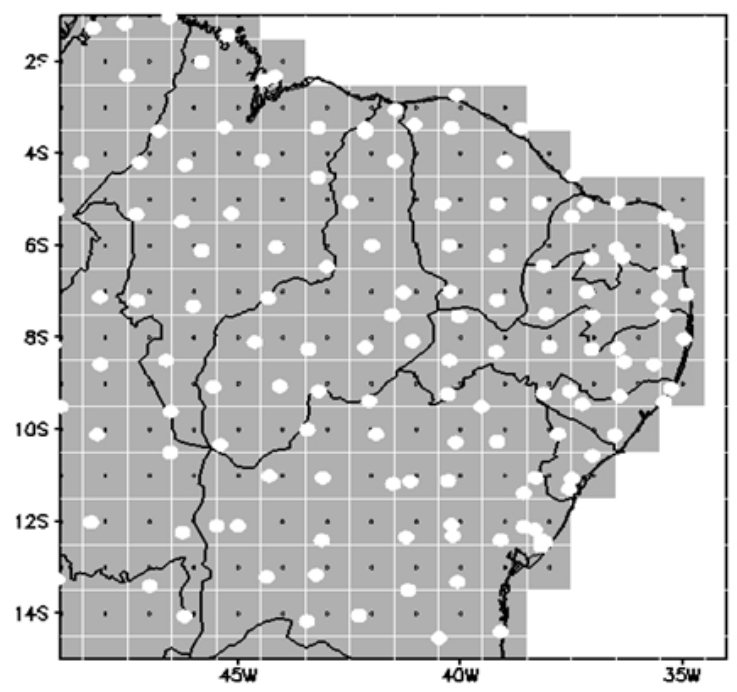

Figura 1 - Estações pluviométricas (pontos em branco) e grade regular de $1^{\circ} \times 1^{\circ}$ (pontos em preto) sobre a região do NEB. O retângulo destacado em linha pontilhada indica o setor norte do Nordeste (SNNEB) usada na investigação do sinal intrassazonal. temperatura do ar nos níveis de pressão e pressão a superfície foram assimiladas em todo o domínio a cada 6 horas (Sun et al. 2005). Como condição de contorno inferior utilizou-se a Temperatura da Superfície do Mar (TSM) mensal observada do conjunto de dados denominado de optimum interpolation (Reynolds e Smith , 1994).

\subsection{Detecção da variabilidade intrassazaonal}

Para investigar os sinais de variabilidade intrassazonal sobre o NEB aplicou-se a transformada wavelet ou ondeleta, uma metodologia estatística espectral conhecidamente eficaz na detecção objetiva dos principais modos de variabilidade temporal presentes numa série de dados não estacionária (Lau e Weng, 1995; Vitorino et al., 2005). No presente estudo, usou-se a transformada de ondeleta com a função complexa de Morlet, a qual foi aplicada nas séries temporais diárias da precipitação observada e simulada do SNNEB. Matematicamente, a função ondeleta, na escala a e posição b, é dada por:

$$
\Psi_{\mathrm{a}, \mathrm{b}}(t)=\frac{1}{2} \psi\left(\frac{t-a}{b}\right)
$$

onde $\mathrm{a}$ e $\mathrm{b}$ são reais e $\mathrm{a}>0$. A transformada de ondeleta é definida por:

$$
W_{\psi} f(\mathrm{a}, \mathrm{b})=\frac{1}{\sqrt{a}} \int f(t) \psi\left(\frac{t-b}{a}\right) \mathrm{dt}
$$

onde $\mathrm{f}(\mathrm{t})$ é uma função temporal qualquer. A função transformada de Morlet foi dada por:

$$
\Psi_{\mathrm{a}, \mathrm{b}}(t)=\frac{1}{\sqrt{\pi}} \ell^{\mathrm{i} \omega_{0} \mathrm{t} \ell^{\frac{-t^{2}}{2}}}
$$

onde $\omega_{0}=6$. As análises espectrais foram avaliadas com base na decomposição em tempo e frequência multi escala no escalograma contendo o espectro de energia da ondeleta, destacando-se os picos estatisticamente significativos ao longo da série temporal. O espectro de potência da ondeleta é o quadrado do valor absoluto da transformada da ondeleta, que indica a variação da série de dados usada, em cada escala de tempo. O tempo médio do espectro de potência da ondeleta dos valores de precipitação resulta de um espectro global de energia da ondeleta, que pode ser usado para avaliar as mudanças nãoestacionárias na variância dos dados (Souza e Ambrizzi, 2006). Considerou-se o espectro de Fourier com ruído vermelho, e a distribuição qui-quadrado foi usada para calcular a significância estatística dos picos espectrais ao nível de 95\%. A formulação matemática encontra-se detalhada em Torrence e Compo (1998).

Além da análise das séries temporais para todo o período análises específicas para composições de anos com contrastes 
climáticos (listados na Tabela 1), definidos conforme Alves et al. (2007) também foram executadas.

Não existe uma definição universal do fenôneno El Nino-Oscilação Sul - ENOS (Scor, 1983, Trenberth, 1997, Chen et al., 2002). Na maioria das vezes este fenômeno acoplado oceano-atmosfera é definido pelo Índice de Oscilação Sul (IOS), dado pela diferença da pressão ao nível médio do mar entre Tahiti e Darwin (e.g., Ropelewski e Jones 1987) e os índices de monitoramento do Pacífico equatorial, dado pelas anomalias de TSM registradas nas regiões conhecidas como Niño3 $\left(5^{\circ} \mathrm{N}-5^{\circ} \mathrm{S}, 150^{\circ} \mathrm{W}-90^{\circ} \mathrm{W}\right)$ e Niño3.4 $\left(5^{\circ} \mathrm{N}-5^{\circ} \mathrm{S}\right.$, $\left.170^{\circ} \mathrm{W}-120^{\circ} \mathrm{W}\right)$, conforme Trenberth (1997). Baseado em análises anteriores, optou-se pelo uso do Niño3, área que é mais correlacionada com o clima do Nordeste (Uvo et al., 1998). Neste estudo utilizou-se uma adaptação da definição proposta por Trenberth (1997). Episódios no Pacífico foram definidos como positivo (PacPos: El Niño) e negativo (PacNeg: La Niña), quando a anomalia de TSM no Niño3, em média, para o período de Novembro a Janeiro é superior a $0,5^{\circ} \mathrm{C}$ e inferior a $-0,5^{\circ} \mathrm{C}$, respectivamente, enquanto nos episódios neutros ( $\mathrm{PacNeu}$ ) a anomalia de TSM permanece entre $-0,5^{\circ} \mathrm{C}$ e $0,5^{\circ} \mathrm{C}$. Os meses de Novembro a Janeiro foram escolhidos em função de representarem melhor a fase de maturação do El Niño/La Niña, e porque incluem um possível atraso de 3 meses entre a conexão atmosférica do Pacífico e o Atlântico tropical (Enfield e Mayer, 1997; Saravanan e Chang, 2000), com possíveis impactos na estação chuvosa do Nordeste do Brasil (Souza et al. 2004).

Não obstante, no Oceano Atlântico o mecanismo dominante no outono austral é o dipolo ou gradiente de anomalias de TSM no setor intertropical (Nobre e Shukla, 1996; Souza, 1997). Este dipolo associa-se com a atuação da ZCIT ao sul do Equador, quando da ocorrência da sua fase negativa, i.e., concomitância de anomalias negativas/positivas de TSM na bacia norte/sul do Atlântico, explicando as condições de chuva acima do normal no NEB (Souza et al., 2004). A fase positiva do dipolo, anomalias positivas/negativas de TSM na bacia norte/sul do Atlântico, relaciona-se com a atuação da ZCIT ao norte do Equador, explicando condições de redução de chuva no NEB (Nobre e Shukla, 1996). Assim sendo, para este trabalho considera-se dipolo positivo (DipPos) e dipolo negativo (DipNeg), quando a diferença nas anomalias de TSM da bacia norte $\left(5^{\circ} \mathrm{N}-20^{\circ} \mathrm{N}\right.$ e $\left.60^{\circ} \mathrm{W}-30^{\circ} \mathrm{W}\right)$ e da bacia sul $\left(5^{\circ} \mathrm{S}-20^{\circ} \mathrm{S}\right.$ e $30^{\circ} \mathrm{W}-10^{\circ} \mathrm{W}$ ) apresentam a média de fevereiro a abril superior a $0,2^{\circ} \mathrm{C}$ e inferior a $-0,2^{\circ} \mathrm{C}$, respectivemente. Dipolo neutro ocorre quando a diferença permaneceu dentro de $\pm 0,2^{\circ} \mathrm{C}$.

A Tabela 1 mostra os eventos (classificados segundo as definições mencionadas) para as fases do ENOS no Pacífico e do Dipolo no Atlântico. As composições para cada evento foram utilizadas para investigar o sinal intrassazonal da chuva observada e simulada no SNNEB.

\section{RESULTADOS E DISCUSSÕES}

\subsection{Climatologia sazonal}

Considerando a média sazonal entre os meses de Fevereiro, Março e Abril (FMA), período em que se verifica o pico da estação chuvosa sobre o SNNEB, observa-se na evolução interanual dos desvios normalizados de precipitação observada (OBS) e simulada (RSM), uma correspondência bastante estreita durante o período de 1974 a 2000, com correlação linear da ordem de 0,84 explicando em torno de 70\% da variância das observações (Figura 2). Portanto, em termos do sinal sazonal, nota-se que o RSM simula muito bem os valores observados sobre a região do SNNEB. Ressalta-se que, como as simulações utilizaram a TSM média mensal observada, era de se esperar esta consistente resposta sazonal da precipitação simulada em relação à observada, visto que os padrões de TSM, principalmente, nos oceanos Atlântico e Pacífico Tropicais tem grande influência na precipitação do SNNEB.

A Tabela 2 sumariza os resultados da precipitação sazonal de FMA, observada e simulada, para composições dos eventos climáticos de grande escala relacionados às fases do ENOS no Pacífico e do Dipolo no Atlântico intertropical. Em geral, nota-se na maioria dos eventos que a precipitação média do RSM apresentou-se bem próxima da OBS, sendo que os respectivos desvios normalizados e percentuais são

Tabela 1 - Classificação dos eventos climáticos associados a fases de aquecimento, resfriamento e neutro no Pacífico e Atlântico, durante o período de 1974-2000.

\begin{tabular}{ll}
\hline Cenários climáticos de grande escala & Anos de ocorrência \\
DipNeg/PacNeg & $1974,1984,1985,1986,1989$ e 2000 \\
DipNeg/PacNeu & 1991 e 1994 \\
DipNeg/PacPos & 1988 e 1995 \\
DipNeu/PacNeg & $1975,1976,1996$ e 1999 \\
DipNeu/PacNeu & 1982,1990 e 1993 \\
DipNeu/PacPos & 1977,1987 e 1998 \\
DipPos/PacNeg & 1997 \\
DipPos/PacNeu & $1978,1979,1980$ e 1981 \\
DipPos/PacPos & 1983 e 1992 \\
\hline
\end{tabular}




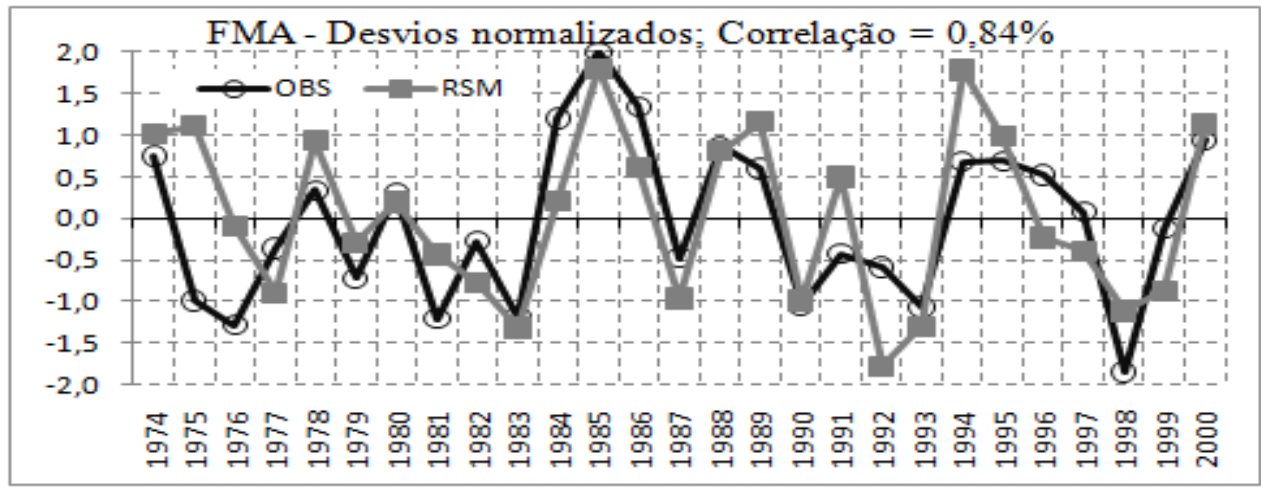

Figura 2 - Desvios normalizados de precipitação (desvio padrão) sazonal para o período de Fevereiro a Abril (FMA) considerando os dados observados (OBS) e simulados (RSM) sobre o SNNEB, durante os anos de 1974 a 2000.

perfeitamente consistentes no que se refere ao sinal negativo ou positivo, dependendo do evento climático em questão. Entretanto, a magnitude dos desvios apresenta diferenças entre OBS e RSM (Tabela 2).

Considerando-se os eventos climáticos de DipNeg com PacNeg (La Niña), ou seja, Pacífico e Attântico favoráveis à chuva no NEB, verifica-se precipitação sazonal acima do normal (desvios positivos), com percentuais de $+21 \% \mathrm{e}+17 \%$ para OBS e RSM, respectivamente, ou seja, muito próximos.

Nas condições de Atlântico favorável com DipNeg e o Pacífico neutro (PacNeu) tem-se desvios positivos de chuva, com percentuais em de 2,3\% para OBS e $11 \%$ para o RSM. Mantendo o Atlântico favorável com DipNeg, porém com o Pacífico desfavorável (PacPos), ainda observam-se desvios positivos de chuva sazonal, com percentuais observados e simulados bastante próximos de $14 \%$.

Durante os anos com eventos neutros sobre o Atlântico intertropical (DipNeu) intercalados com as condições de Pacífico neutro (PacNeu), favorável (PacNeg) e desfavorável (PacPos) notam-se desvios negativos de precipitação sazonal, com percentuais variando de $-14,5 \%,-1,5 \% \mathrm{e}-15 \%$, respectivamente, na simulação do RSM.
Quando as condições do Atlântico encontram-se desfavoráveis (DipPos) e do Pacífico favoráveis (PacNeg), notam-se desvios e percentuais de precipitação próximos de zero, sugerindo que uma condição de grande escala pode anular a outra. Entretanto, mantendo o Atlântico desfavorável com o Pacífico neutro (PacNeu), tem-se desvios negativos de chuva e percentuais que alcançam $-9 \%$ no OBS e $-0,4 \%$ no RSM. Por outro lado, ambas as condições desfavoráveis do Atlântico (DipPos) e Pacífico (PacPos) associam-se com desvios negativos de chuva com percentuais em torno de $-20 \%$ nos dados observados e $-23,5 \%$ no RSM.

\subsection{Variabilidade intrassazonal}

A seguir, investiga-se o sinal intrassazonal sobre o SNNEB, com base na análise de ondeletas aplicada à precipitação diária (01 de Janeiro a 30 de Junho) dados observados e simulados, considerando diferentes eventos climáticos de grande escala. Tais análises foram enfatizadas para os eventos que apresentaram um desvio percentual em módulo maior que 14\% (DipNeg/PacNeg, DipPos/PacPos, DipNeu/ PacPos e DipNeg/PacPos).

Tabela 2 - Média das composições (mm), desvio normalizado (desvio padrão) e desvio percentual (\%) da precipitação sazonal em FMA observada (OBS) e simulada (RSM) sobre o SNNEB correspondente aos diferentes eventos definidos na Tabela 1. Valores em negrito indicam anomalias significantes em $90 \%$ segundo test-t de student.

\begin{tabular}{lllllllllll}
\hline & & $\begin{array}{l}\text { DipNeg/ } \\
\text { PacNeg }\end{array}$ & $\begin{array}{l}\text { DipNeg/ } \\
\text { PacNeu }\end{array}$ & $\begin{array}{l}\text { DipNeg/ } \\
\text { PacPos }\end{array}$ & $\begin{array}{l}\text { DipNeu/ } \\
\text { PacNeg }\end{array}$ & $\begin{array}{l}\text { DipNeu/ } \\
\text { PacNeu }\end{array}$ & $\begin{array}{l}\text { DipNeu/ } \\
\text { PacPos }\end{array}$ & $\begin{array}{l}\text { DipPos/ } \\
\text { PacNeg }\end{array}$ & $\begin{array}{l}\text { DipPos/ } \\
\text { PacNeu }\end{array}$ & $\begin{array}{l}\text { DipPos/ } \\
\text { PacPos }\end{array}$ \\
\hline Média & OBS & 8,1 & 6,9 & 7,6 & 6,1 & 5,9 & 5,6 & 6,8 & 6,1 & 5,4 \\
composição & RSM & 7,7 & 7,3 & 7,5 & 6,5 & 5,6 & 5,6 & 6,7 & 6,6 & 5,0 \\
\hline \multirow{2}{*}{$\begin{array}{l}\text { Desvio } \\
\text { normalizado }\end{array}$} & OBS & $\mathbf{1 , 2}$ & 0,1 & $\mathbf{0 , 8}$ & $\mathbf{- 0 , 5}$ & $-0,6$ & $\mathbf{- 0 , 9}$ & 0,1 & $\mathbf{- 0 , 5}$ & $\mathbf{- 1 , 1}$ \\
\hline Resvio & OBS & $\mathbf{1 , 0}$ & 0,6 & $\mathbf{0 , 8}$ & $-0,2$ & $\mathbf{- 1 , 0}$ & $\mathbf{- 1 , 0}$ & 0,1 & $-0,1$ & $\mathbf{- 1 , 6}$ \\
percentual & RSM & 17,0 & 11,2 & 13,6 & $-1,5$ & $-14,5$ & $-15,1$ & 1,6 & $-0,4$ & $-23,5$ \\
\hline
\end{tabular}


A Tabela 2 mostra que as situações extremas de precipitação sobre o SNNEB, com aumento de $+21 \%$ (diminuição de -23\%) no total de chuva sazonal de FMA, associam-se as condições favoráveis (desfavoráveis) do Pacífico e Atlântico, ou seja, o cenário DipNeg/PacNeg (DipPos/PacPos), e situações com impactos menores na precipitação do SNNEB relativos a composição de anos DipNeu/PacPos e DipNeg/ PacPos, com desvios de $-16 \%$ e $+14 \%$, respectivamente.

A Figura 3 ilustra o escalograma da energia da ondeleta obtida para os dados diários de chuva correspondentes aos anos individuais de ocorrência do DipNeg/PacNeg, bem como para a média da composição. Observa-se que o RSM captura qualitativamente a configuração do sinal espectral a partir de um período de oscilações de precipitação diária acima de oito dias. As oscilações e suas significâncias de mais altas frequências (período menor do que 8 dias), na precipitação diária sobre SNNEB, não foram bem reproduzidas pelo RSM. Estas frequências estão associadas a distúrbios de escala sinótica do tipo influências de sistemas frontais no setor centro-sul do NEB, atuação de vórtices ciclônicos e cavados de altos níveis, que causam precipitação no setor norte do NEB e áreas sujeitas a complexos convectivos de mesoescalas e circulações locais tipo brisas comuns nas regiões litorâneas do NEB. Ao longo de quase todos os anos com DipNeg/ PacNeg, os picos de maior energia espectral entre 32 dias, 64 dias e 128 dias, também tiveram boa similaridade entre OBS e RSM, ressaltando maior similaridades nos anos de 1974, 1985 e 2000. Considerando a média da composição para a anos de DipNeg/
PacNeg, o RSM capturou a configuração do espectro de energia da precipitação diária no SNNEB e sua significância estatística (último quadro do lado direito da Figura 3). As maiores energias espectrais, nos primeiros 100 dias (aproximadamente entre janeiro e março) concentraram-se nas escalas entre 8 e 64 dias. A partir de março até junho, maior energia espectral é encontrada na escala intrassazonal, entre 64 e 128 dias.

A Figura 4 apresenta o escalograma da energia da ondeleta durante eventos DipPos/PacPos. Em geral, a simulação do RSM para os anos de 1983, 1992 obteve bom desempenho ao simular espectro de energia da chuva diária no período superior a 8 dias similar ao OBS. Picos de energia espectral no período de 16 e 32 dias, entre janeiro e o final de março (tempo entre 01 e 100 dias) no ano de 1983, e em torno do período de 32 dias, de janeiro ao final de fevereiro, nos anos de 1992 foram bem similares as observações. Os picos espectrais de energia, observados no período entre 32 e 64 dias e entre 64 e 125 dias no ano de 1992, não foram bem simulados em intensidade pelo RSM, assim como as oscilações de altas frequências tanto em 1983 como em 1992. A configuração espectral da composição média, a despeito da utilização de apenas dois anos, não foi bem capturada como ocorreu na composição do cenário DipNeg/ PacNeg. Uma similaridade foi observada para os períodos de maior energia espectral, que ocorreram na escala entre 64 e 128 dias, principalmente nos primeiros dias do ano.

Outro cenário com impacto negativo $(-16 \%$ de diminuição no total sazonal) na chuva do SNNEB ocorreu
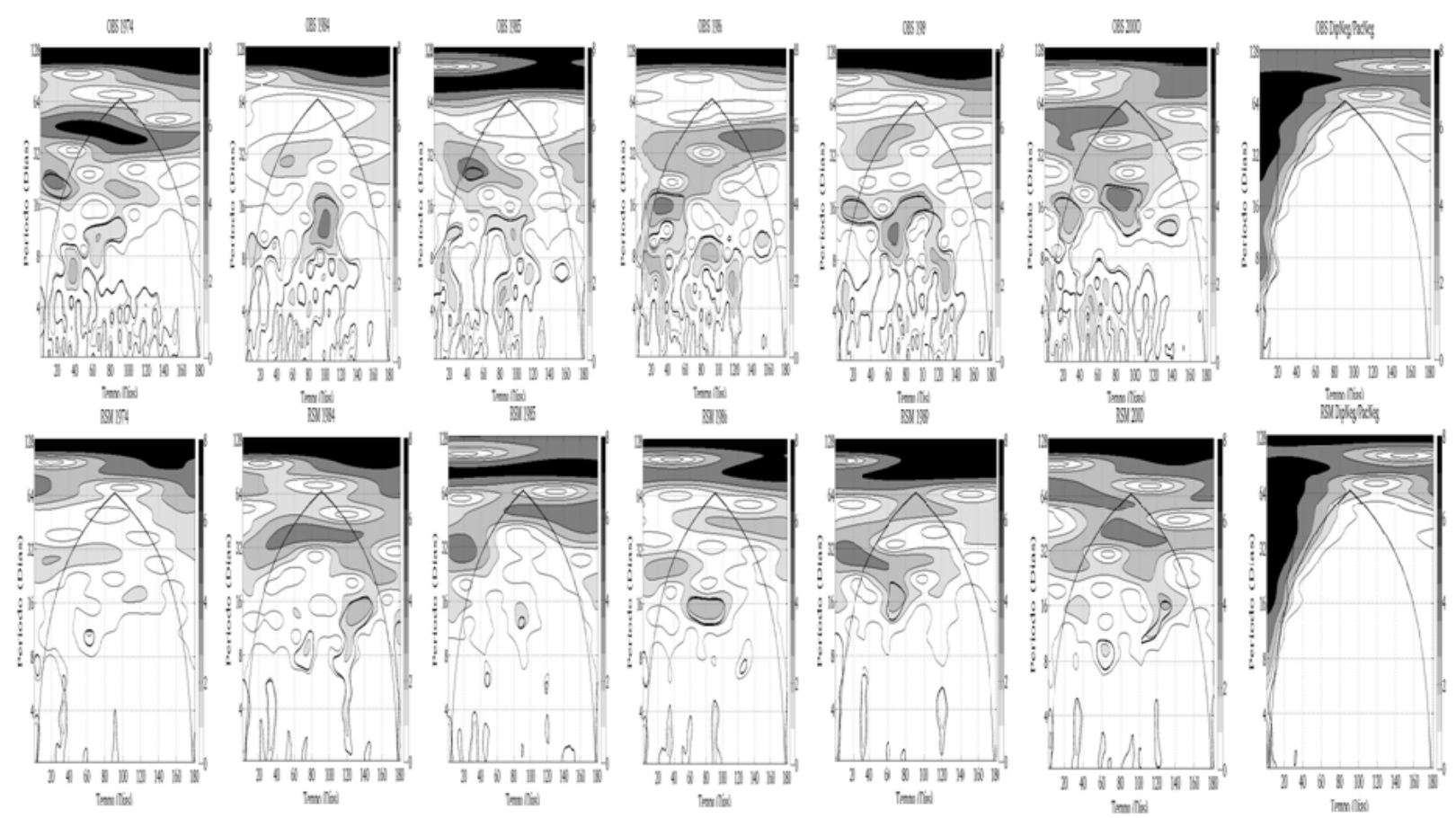

Figura 3 - Escalograma de energia da ondeleta $\left(\mathrm{mm}^{2}\right)$ da precipitação no período chuvoso (181 dias: 1 Janeiro a 30 Junho) para os dados observados (OBS) e simulados (RSM), correspondentes aos anos com o cenário DipNeg/PacNeg. A isolinha contínua mais grossa é o nível de significância em $5 \%$. A linha fina contínua em forma cônica indica o cone de influência. 

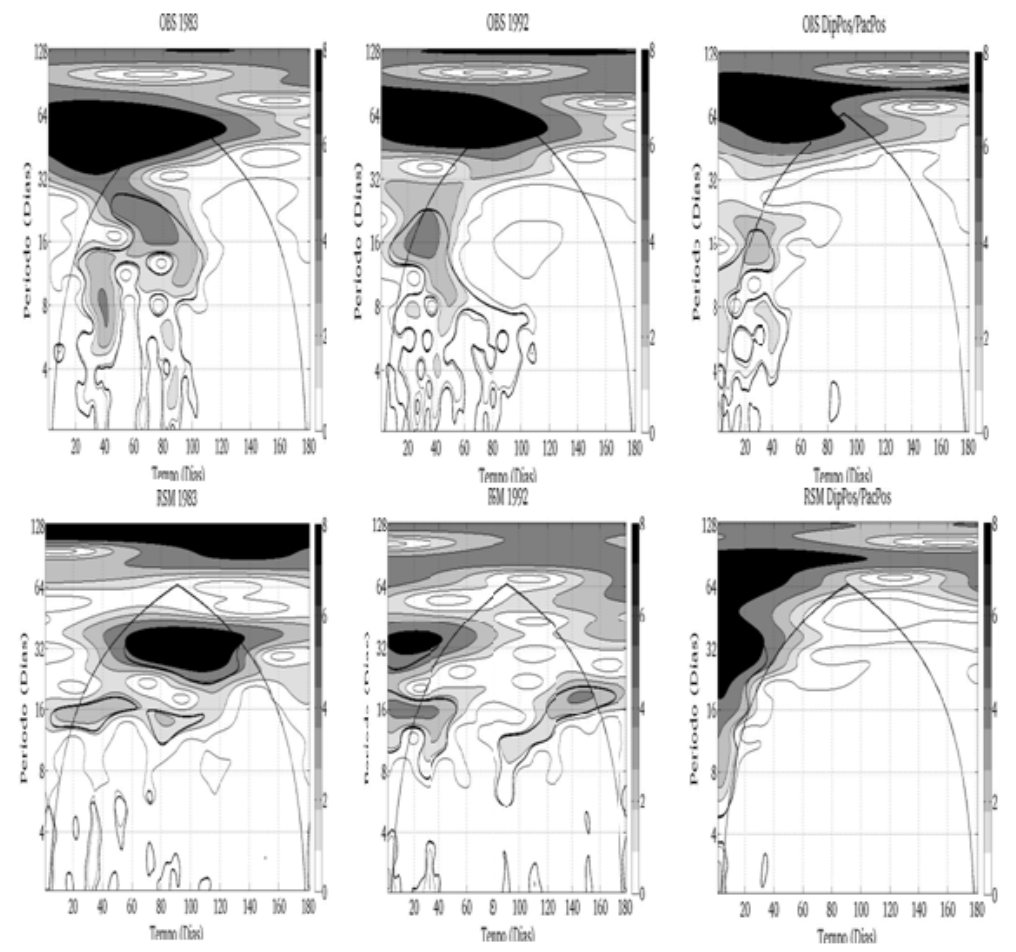

Figura 4 - Idem a Figura 3, porém para os anos com o cenário DipPos/PacPos.

nas condições de DipNeu/PacPos, ou seja, Atlântico neutro com Pacífico desfavorável (El Niño). Para este cenário, a Figura 5 mostra, como já identificado nas Figuras 3 e 4, que o downscaling com o RSM identificou a variabilidade espectral observada no SNNEB, principalmente a partir de períodos acima de 8 dias. A energia espectral e sua significância estatística na chuva diária observada no SNNEB em períodos de mais alta frequência (inferior a 8 dias), não foi simulada pelo RSM. Os espectros de energia contidos na chuva diária observada foram bem simulados na escala de tempo entre 16-32 dias, entre o início de janeiro e final de junho nos dois anos da composição, mais evidentes entre 01 de janeiro e aproximadamente final de março (tempo de 01 a 100 dias).

Como para as composições analisadas anteriormente, a configuração média para o cenário DipNeu/PacPos mostrou uma configuração parecida a da precipitação diária observada no SNNEB (último quadro do lado direito da Figura 5). Os maiores picos energéticos concentraram-se nas escalas intrassazonais (em torno de 32 e 64 dias) entre 01 de janeiro e final de março, sendo também simuladas pelo RSM. Oscilações de precipitação com períodos superior a 64 dias nos seis primeiros meses do ano, com relevância espectral, também tiveram boa representação no RSM.

$\mathrm{E}$, finalmente, outro cenário com impacto positivo no regime de chuva do SNNEB ocorre nas condições de DipNeg/ PacPos, ou seja, Atlântico favorável com Pacífico desfavorável
(El Niño). Para este cenário, a Figura 6 mostra, tanto nos anos individuais como na média da composição, ressaltando que apenas dois anos foram usados para média, características similares, evidenciando que o RSM captura o sinal intrassazonal de precipitação nos períodos acima de 8 dias, pelo menos qualitativamente. Há uma boa concordância entre os picos espectrais de precipitação observados nos períodos entre 16-64 dias e simulados pelo RSM (Figura 6). As oscilações espectrais de alta frequência, para períodos inferiores a 8 dias, e suas significâncias estatísticas não foram capturadas pelo RSM, como já evidenciado para os outros eventos climáticos. As configurações médias da composição observada e a simulada pelo RSM, nestes anos de DipNeg/PacPos, foram bem consistentes na estrutura qualitativa da distribuição espectral da chuva diária no SNNEB. Uma característica comum, independente do cenário e da variabilidade interanual, é que em média, com também já mostrado na composição dos outros cenários, há um predomínio de maiores picos espectrais de energia entre o primeiro e o centésimo dia do ano com ocorrências de precipitação em períodos que variaram de 8 a 64 dias. Esta característica foi bem simulada qualitativamente pelo RSM.

Uma característica observada nas análises anteriores para as composições dos eventos é o fraco desempenho das simulações do RSM em reproduzir a significância estatística dos eventos de precipitação observados de alta frequência, com 

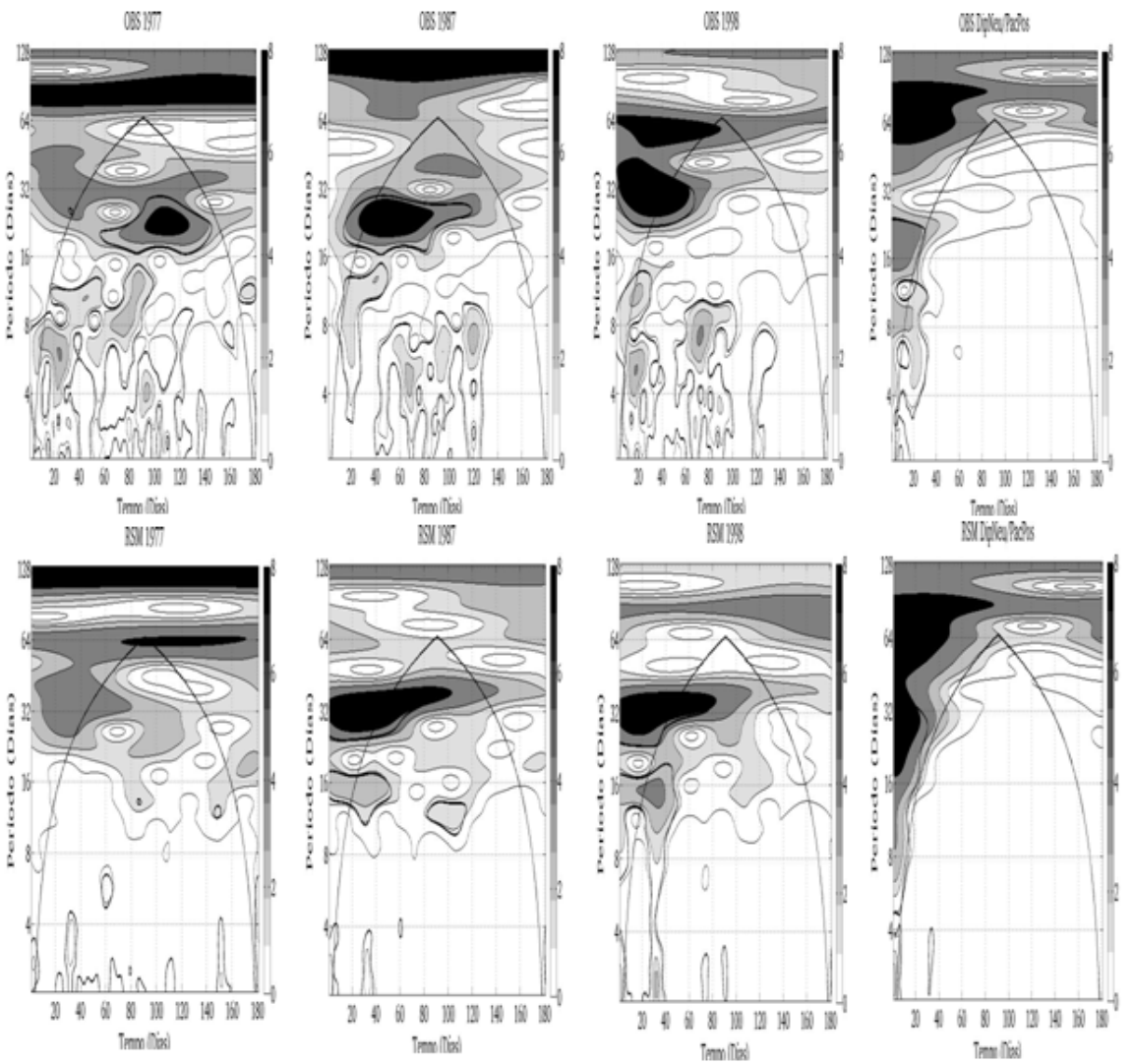

Figura 5 - Idem a Figura 3, porém para os anos com o cenário DipNeu/PacPos.

período menor do que 8 dias. Presumem-se que duas possíveis causas podem estar associadas a esta característica. A primeira é que o RSM tem menor variância nas intensidades diárias de precipitação do que a precipitação observada para períodos abaixo da escala de 8 dias. A outra seria devido ao processo de simulação do RSM que foi para fins climáticos. Neste caso cada inicialização do modelo foi feita apenas uma vez com condições da atmosfera antes do dia 01 de janeiro de cada ano no período de 1974-2003, o que deixa de ser importante na captura das variações de precipitação associados à escala sinótica ao longo do período sazonal de simulação. Vale mencionar também a quase inexistência de estudos que possibilitem comparar os resultados aqui apresentados, pois existem poucas comparações entre resultados de modelagem climática com uso de downscaling na variabilidade da escala intrassazonal em particular para o SNNEB (Alves et al. 2008; Lima e Alves, 2009). Pezzi e Kayano (2009) usando resultados do modelo de circulação geral do Centro de Previsão de Tempo e Estudos Climáticos (CPTEC), com várias parametrizações convecção, mostraram que a técnica de ondeletas tem potencial para identificar a variabilidade interanual do espetro de precipitação mensal em várias áreas da América do Sul, porém a intensidade e magnitude do espectro simulado diferem do observado. Entretanto, Pezzi e Kayano (2009) não investigaram o sinal na escala intrassazonal.

\section{CONCLUSÃO E RECOMENDAÇÕES}

Entre as principais conclusões do estudo tem-se que o RSM capturou bem o sinal sazonal da precipitação, em média, sobre o SNNEB com uma explicação da variância do total de chuva observado entre Fevereiro-Abril em torno de $70 \%$ no período de 1974-2000. Para os anos de contrastes climáticos observados nos Oceanos Pacífico e Atlântico Tropicais, os resultados mostraram que o RSM conseguiu também um bom desempenho na estimativa das médias, desvios padrões e percentuais da chuva. Além disso, a análise de ondeletas permitiu evidenciar que o RSM também simulou o sinal intrassazonal da precipitação observada, particularmente as oscilações nas escalas com períodos acima de 8 dias, ao longo do período de 01 de janeiro a 30 de junho, em alguns meses de anos cujos extremos climáticos observados nos oceanos tropicais tiveram um impacto maior na precipitação do SNNEB (DipNeg/PacNeg e DipPos/PacPos), e em anos cujos impactos destes oceanos foram menos intensos na precipitação (DipNeu/ PacPos e DipNeg/PacPos). Ficou evidente que o RSM teve bom desempenho na reprodução do espectro de eventos de precipitação na escala de 64 a 128 dias, observados no período de 01 de janeiro a 30 de junho. Outro resultado é de fraco desempenho, em todos os anos analisados (1974-2000), figuras 

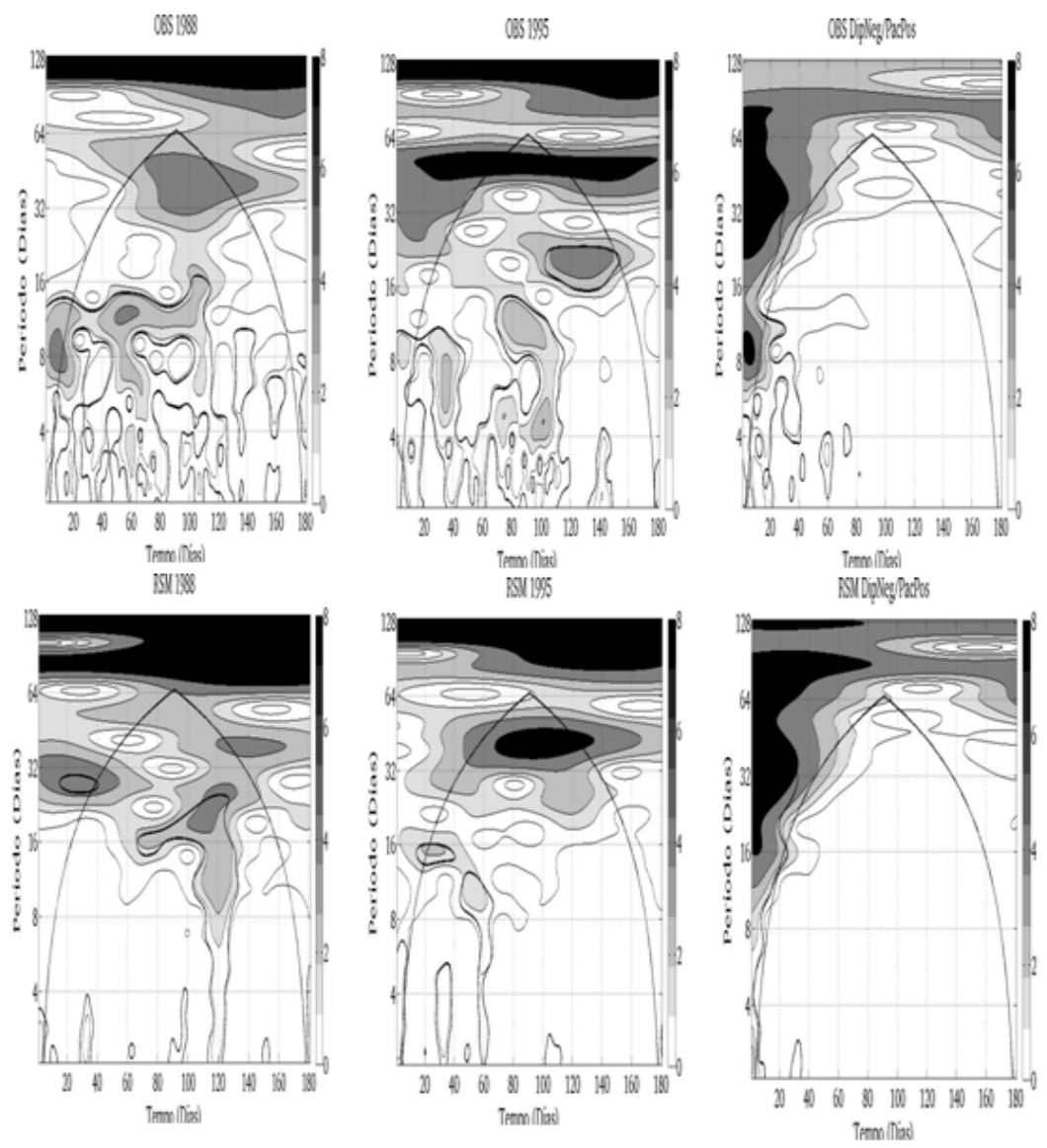

Figura 6 - Idem a Figura 3, porém para os anos com o cenário DipNeg/PacPos.

não mostradas, embora o número de anos seja pequeno para estas composições, do RSM em simular o padrão e a significância estatística dos eventos de precipitação de alta frequência (período menor de 8 dias).

Para estudos futuros recomenda-se uma análise destas características intrassazonais do RSM em modo de previsão, usando-se simulações com a TSM prevista e persistida para se identificar possíveis diferenças entre o modo de previsão e o resultado apresentado aqui em modo de simulação.

\section{REFERÊNCIAS BIBLIOGRÁFICAS}

ALVES, J. M. B.; CAMPOS, J. N. B. NASCIMENTO, L. S. V. Sensibilidade intrasazonal de um downscaling dinâmico de precipitação (1971-2000): Uma análise na bacia hidrográfica do Açude Castanhão-CE. Revista Brasileira de Meteorologia, São Paulo, v.23, n.1, p.73-87, 2008.

ALVES, J. M. B.; CAMPOS, J. N. B.; NASCIMENTO, L. S. V. BARROS, F. V. F. Estudo preliminar intrasazonal do acoplamento entre modelagem dinâmica de precipitação e vazão no Nordeste do Brasil. In: Anais do XVII Simpósio Brasileiro de Recursos Hídricos e $8^{\circ}$ Simpósio de Hidráulica e
Recursos Hídricos de Países de Língua Oficial Portuguesa. São Paulo, Novembro. 2007.

CARVAlHO, L. M. V.; JONES, C. LIEBMANN, B. The South Atlantic Convergence Zone. Intensity, form, persistence, and with intra-seasonal to interannual activity and extreme rainfall. Journal of Climate, Boston, v.17, p.88-108, 2004.

CHEN, C. C.; McCARL, B. A. HIEL, H. S. J. Agricultural value of ENSO information under alternative phase definition. Climate Change, Amsterdan, v.54, p.305-325. 2002.

ENFIELD, D. B. MAYER, D. A. Tropical Atlantic sea surface temperature variability and its relation to El Niño-Southern Oscillation. Journal of Geophysical Research, Boston, v.102:929-945. 1997.

HIGGINS, R. W. SHI, W. Intercomparison of the principal modes of interannual and intraseasonal variability of the North American monsoon system. Journal of Climate, Boston, v.14, p. 403-417. 2001.

JONES, C. CARVALHO, L. M. V. Active and breack phases in the south American monsoon system. Journal of Climate, Boston, v.15, p.905-914. 2002.

JONES, C. Occurrence of extreme precipitation events in California and relationship with the Madden-Julian oscillation. Journal of Climate, Boston, v.13, p.3576-3587. 2000. 
JONES, C.; CARVALHO, L. M. V.; HIOGGINS, R. W.; WALISER, D. E. SCHEMM, J. -K. E. Climatology of tropical intraseasonal convective anomalies: 1979-2002. Journal of Climate, v.17, p. 523-539. 2004.

JUANG, H.-M. H. KANAMITSU, M. The NMC nested regional spectral model. Monthly Weather Review, Boston, v.122, p.326. 1994.

LAU, K.M. WENG H.Y. 1995. Climate signal detection using wavelets transform: how to make a time series sing. Bulletin of American Meteorological Society, Boston, v.76: 2391-2402.

LIMA, J. P. R. ALVES, J. M. B. Um estudo de um downscaling dinâmico de precipitação intrasazonal acoplado a um modelo chuva-vazão na bacia hidrográfica do alto-médio São Francisco. Revista Brasileira de Meteorologia, São Paulo, v.24, n.3, p.323338. 2009.

MADDEN, R. A. JULIAN, P. R. Observations of the 40-50 day tropical oscillation: A review. Monthly Weather Review, Boston, v,112, p.814-837. 1994.

MO, K. C. Intraseasonal modulation of summer precipitation over North America. Monthly Weather Review, Boston, v.128, p. 1490-1505. 2000.

NOBRE, P. MELO, A. B. C. Variabilidade climática intrasazonal sobre o Nordeste do Brasil em 1998-2000. Revista Climálise. Publicação on line. 2001.

NOBRE, P.; MOURA, A. D.; SUN, L. Dynamical downscaling of seasonal climate prediction over Nordeste Brazil with ECHAM3 and NCEP'S Regional Spectral Model at IRI. Bulletin of American Meteorological Society, Boston, v. 82, p. 2787-2796. 2001.

NOBRE, P.; SHUKLA, J. Variations of sea surface temperature, wind stress, and rainfall over the tropical Atlantic and South America, Journal of Climate, Boston, v. 9 n.10, p.2464-2479, 1996.

PAEGLE, J.; BYERLE, L. A. MO, K. Intraseasonal modulation of South American summer precipitation. Monthly Weather Review , Boston, v.128, p. 837-850. 2000.

PEZZY. L. P. KAYANO, M. T. An analysis of seasonal precipitation forecast in South América using wavelets. International Journal of Climatology, Londres, v.29: 1560-1573 (2009). DOI: 10.1002/ joc. 1813 .

RASMUSSON, E. M. ARKIN, P. A. A global view of large-scale precipitation variability. Journal of Climate, Boston, v.6, p. 1495-1522. 1993.

REPELLI, C.; A.; SOUZA, E. B.; QUADRO, M. F. L; ALVES, J. M. B. SAKAMOTO, M. S. O episódio de chuvas intensas no nordeste brasileiro no final de março/97: influência da oscilação 30-60 dias. Revista Brasileira de Meteorologia, São Paulo, 13, p. 09-18. 1998.

REYNOLDS, R. W. SMITH,T. M. Improved global sea surface temperature analysis using optimum interpolation. Journal of Climate, Boston, v.7, p.929-948.1994.
ROECKNER, E. et. al. The atmospheric general circulation model ECHAM4: model description and simulation of the presentday climate. Max Planck Institute für Meteorologie, Report n.28, Hamburg, Germany, 90p. 1996.

ROPELEWSKI, C. F. JONES, C. F. An extension of the Tahiti-Darwin Southern Oscillation index. Monthly Weather Review, Boston, v.115, p.2161-2165. 1987.

SARAVANAN, R. CHANG, P. Interactions between tropical Atlantic variability and El Niño-Southern Oscillation. Journal of Climate, Boston, v.13, p.2195-2216. 2000.

SCOR (Scientific Committee for Ocean Research) (1983) Prediction of El Niño. In: Proc 19th Meet of the Scientific Committee for Ocean Research working group, v.55. SCOR, Paris, p 47-51.

SOUZA, E. B.; KAYANO, M. T. AMBRIZZI, T. The regional precipitation over eastern amazon/northeast Brazil modulated by tropical Pacific and Atlantic SST anomalies on weekly timescale. Revista Brasileira de Meteorologia, v.19, n.2, p.113-122. 2004.

SOUZA, E. B.; AMBRIZZI, T., Modulation of the intraseasonal rainfall over tropical Brazil by the Madden-Julian oscillation. International Journal of Climatology, Londres, v. 26, p. 17591776, 2006

SOUZA, E. B. Um estudo observacional sobre o Padrão de Dipolo de anomalias de TSM no Oceano Atlântico Tropical. 138f. 1997 (Mestrado em Meteorologia) - INPE, São José dos Campos -SP, 1997 (INPE-6392-TDI/608).

SUN, L.; MONCUNILL, D.; HUILAN, L.; MOURA, A. D.; FILHO, F. A. S. Climate downscaling over Nordeste, Brazil, using the NCEP RSM97. Journal of Climate, Boston, v18, n.4, p..551-567. 2005. SUN, L.; MONCUNILL, D.; HUILAN, L.; MOURA, A. D.; FILHO, F. A. S.; ZEBIAK, E. An operational dynamical downscaling prediction system for Nordeste Brazil and the 2002-04 real time forecast evaluation. Journal of Climate, Boston, v19, n.10, p.1990-2007. 2006.

TORRENCE, C; COMPO, G. P. 1998. A practical guide to wavelet analysis. Bulletin of American Meteorological Society, Boston, v.79, n.1, p. 61-78. 1998

TRENBERTH, K. E. The definition of El Niño. Bulletin of American Meteorological Society, Boston, v.78, p.2771-2777. 1997.

UVO, C. B.; REPELLI, C. A.; ZEBIAK, S. E. KUSHNIR, Y. The relationship between tropical Pacific and Atlantic SST and northeast Brazil monthly precipitation. Journal of Climate, Boston, v.11, p.551-562. 1998.

VITORINO, M. I., SILVA DIAS, P. L., FERREIRA, N. J., 2005: Observational study of the seasonality of the submonthly and sntraseasonal signal over the tropics. Journal of Meteorological Atmospheric Physics. 2005 (DOI 10.1007/s00703-005-0162-7).

WEICKMANN, K. M. El Niño/Southern Oscillation and the MaddenJulian (30-60 day) oscillations during 1981-1982. Journal of Geophysical Research, Boston, v.96, p.3187-3195. 1991. 